\title{
Incidence de la COVID-19 sur les issues de grossesse : examen systématique et méta-analyse
}

\author{
Shu Qin Wei MD PhD, Marianne Bilodeau-Bertrand MSc, Shiliang Liu MD PhD, Nathalie Auger MD MSc
}

Citation : CMAJ 2021 April 19;193:E540-8. doi : 10.1503/cmaj.202604-f; diffusion hâtive le 19 mars 2021

Voir la version anglaise de l'article ici : www.cmaj.ca/lookup/doi/10.1503/cmaj.202604

\section{RÉSUMÉ}

Contexte : La nature exacte des répercussions de la maladie à coronavirus 2019 (COVID-19) sur la santé maternelle et néonatale reste à préciser. Nous avons cherché à évaluer l'association entre l'infection par le coronavirus du syndrome respiratoire aigu sévère 2 (SRAS-CoV-2) pendant la grossesse et les issues défavorables de la grossesse.

MÉTHODES : Nous avons réalisé une revue systématique et une méta-analyse d'études observationnelles fournissant des données comparatives sur l'infection par le SRAS-CoV-2 et la gravité de la COVID-19 pendant la grossesse. Nous avons sélectionné les études admissibles à partir des bases de données MEDLINE, Embase, ClinicalTrials.gov, medRxiv et Cochrane au 29 janvier 2021, en utilisant les Medical Subject Headings (vedettes matière en médecine) et les expressions clés « severe acute respiratory syndrome coronavirus 2 OR SARS-CoV-2 OR coronavirus disease 2019 OR COVID-19 » (coronavirus du syndrome respiratoire aigu sévère 2 ou SRAS-CoV-2 ou maladie à coronavirus 2019 ou COVID-19) AND « pregnancy » (grossesse). Nous avons ensuite évalué la qualité méthodologique de toutes les études retenues avec l'échelle de Newcastle-Ottawa. Les issues primaires étaient la prééclampsie et la naissance prématurée. Les issues secondaires incluaient la mortinaissance et le diabète gestationnel, ainsi que d'autres issues de grossesse. Nous avons calculé des rapports de cotes (RC) sommaires ou des différences moyennes pondérées avec des intervalles de confiance (IC) à $95 \%$ par méta-analyse à effets aléatoires.

RÉSULTATS : Nous avons retenu 42 études portant sur 438548 personnes enceintes. Comparativement à une absence d'infection par le SRAS-CoV-2 pendant la grossesse, le diagnostic de COVID-19 a été associé à la prééclampsie ( $R C$ 1,33; IC à $95 \%$ 1,03-1,73), à la naissance prématurée ( $\mathrm{RC} 1,82$; IC à $95 \% 1,38-2,39)$ et à la mortinaissance (RC 2,11; IC à $95 \%$ 1,14-3,90). Par rapport à la COVID-19 légère, la COVID-19 grave était fortement associée à la prééclampsie (RC 4,16; IC à $95 \%$ 1,55$11,15)$, à la naissance prématurée (RC 4,29; IC à 95\% 2,41-7,63), au diabète gestationnel (RC 1,99; IC à $95 \% 1,09$ $3,64)$ et au faible poids à la naissance (RC 1,89; IC à $95 \%$ 1,14-3,12).

INTERPRÉTATION : La COVID-19 pourrait être associée à un risque accru de prééclampsie, de naissance prématurée et d'autres issues défavorables de la grossesse.
L

a maladie à coronavirus 2019 (COVID-19), causée par le coronavirus du syndrome respiratoire aigu sévère 2 (SRAS-

CoV-2), a fait l'objet d'une déclaration de pandémie en mars 20201. Les personnes enceintes et les nourrissons pourraient être particulièrement susceptibles de contracter la COVID19, car la grossesse induit des changements physiologiques touchant l'appareil cardiorespiratoire et le système immunitaire, ce qui pourrait altérer la réponse à l'infection par le SRAS-CoV- $2^{2}$. Par ailleurs, le fœtus peut être exposé au SRAS-CoV-2 pendant une période critique de son développement ${ }^{3}$. La nature de l'association entre la COVID-19 et les issues de grossesse reste mal définie, et les méta-analyses portant sur des patientes enceintes atteintes de la COVID-19 ont une portée limitée. Quant aux revues publiées, elles se concentrent principalement sur des estimations de prévalence tirées d'études de cas ou de séries de cas difficiles à interpréter et potentiellement biaisées ${ }^{4,5}$. Selon une revue systématique publiée en 2020, les personnes enceintes ne présentaient pas un risque supérieur d'infection par le SRAS-CoV-2 ou de COVID-19 symptomatique, mais elles étaient exposées à un risque accru de COVID-19 grave par rapport à celles qui n'étaient pas enceintes ${ }^{5}$. Notons toutefois que cette revue incluait des cas probables de COVID-19 en plus des 
cas confirmés ${ }^{5}$. Certaines études observationnelles récentes semblent indiquer que les personnes présentant une COVID-19 asymptomatique ou symptomatique confirmée ${ }^{6-15}$, ainsi que des infections légères et graves ${ }^{6,8,9,15-22}$, pourraient être exposées à un risque d'issues de grossesse défavorables, nous n'avons trouvé aucune revue systématique faisant une analyse exhaustive de ces données.

Nous avons réalisé une revue systématique et méta-analyse des issues maternelles, fœtales et néonatales chez les personnes enceintes atteintes de la COVID-19 pour déterminer s'il y a une association entre l'infection par le SRAS-CoV-2 et les issues de grossesse défavorables, notamment la prééclampsie, la naissance prématurée et la mortinaissance.

\section{Méthodes}

Nous avons procédé à une revue systématique selon les directives PRISMA (Preferred Reporting Items for Systematic Reviews and Meta-Analyses).

\section{Sources des données, stratégie de recherche et sélection des études}

Nous avons procédé à une recherche systématique dans les bases de données MEDLINE, Embase, ClinicalTrials.gov, medRxiv et Cochrane afin de recenser les études observationnelles publiées au 29 janvier 2021 comportant des données comparatives sur les personnes atteintes de la COVID-19 qui étaient enceintes (annexe 1, accessible en anglais au www.cmaj.ca/lookup/ doi/10.1503/cmaj.202604/tab-related-content). Notre stratégie de recherche suivait les directives du Peer Review of Electronic Search Strategies (PRESS). Nous avons effectué des recherches dans les bases de données à l'aide d'une combinaison de Medical Subject Headings (vedettes matière en médecine) et des expressions clés " severe acute respiratory syndrome coronavirus 2 OR SARS-CoV-2 OR coronavirus disease 2019 OR COVID-19 » (coronavirus du syndrome respiratoire aigu sévère 2 ou SRAS-CoV-2 ou maladie à coronavirus 2019 ou COVID-19) AND " pregnancy » (grossesse), en restreignant le paramètre linguistique aux résumés en anglais. Nous avons également effectué une recherche manuelle dans les références citées pour ces articles afin d'inclure toute autre étude répondant à nos critères.

Deux chercheuses ont examiné indépendamment les titres et les résumés recensés, comparé les études sélectionnées et résolu toute divergence par une discussion.

Nous avons examiné et sélectionné les articles complets des études évaluant les issues de grossesse maternelles, fœtales et néonatales associées à la COVID-19 qui répondaient aux critères d'inclusion suivants : étude observationnelle, population comprenant des personnes enceintes, infection par le SRAS-CoV-2 confirmée par un test d'amplification en chaîne par polymérase (PCR) ou par des codes pour les cas confirmés de COVID-19 de la Classification statistique internationale des maladies et des problèmes de santé connexes, $10^{e}$ révision (CIM-10), comparaisons comprenant des patientes atteintes de la COVID-19 par rapport à celles qui ne l'étaient pas, patientes atteintes d'une COVID-19 symptomatique par rapport à celles atteintes d'une COVID-19 asymptomatique, ou d'une COVID-19 grave par rapport à une forme légère. Les issues comprenaient la morbidité et la mortalité maternelles, fœtales ou néonatales, l'accès aux données comparatives nécessaires au calcul de la taille de l'effet et un risque de biais faible ou modéré selon les critères d'évaluation de la qualité méthodologique de l'échelle de Newcastle-Ottawa ${ }^{23}$.

Nous avons exclu les études qui répondaient à au moins un des critères suivants : revues, études de cas ou séries de cas, études sans données comparatives, et études incluant des cas de pneumonie infectieuse causés par d'autres agents viraux. Dans les cas où plus d'une étude publiée portait sur la même cohorte et rapportait des résultats identiques, nous avons inclus celle qui contenait les données les plus complètes afin d'éviter les doublons.

\section{Définition de cas}

Nous avons défini les cas de COVID-19 comme une infection par le SRAS-CoV-2 confirmée chez une personne enceinte. Les témoins sans COVID-19 comprenaient les personnes enceintes dont le résultat du test de dépistage par PCR était négatif, celles qui étaient enceintes avant la pandémie ou celles qui étaient enceintes et asymptomatiques au début de la pandémie. Les cas de COVID-19 asymptomatique incluaient les personnes ayant obtenu un résultat positif au test de dépistage du SRAS-CoV-2, mais n'ayant jamais développé de symptômes de la COVID-19, et les cas de COVID-19 symptomatique, les personnes ayant obtenu un résultat positif au test de dépistage du SRAS-CoV-2 qui ont eu de la fièvre, une toux, de l'essoufflement, de la fatigue ou une perte du goût ou de l'odorat. La COVID-19 grave se caractérisait par une dyspnée, une fréquence respiratoire de 30 respirations par minute ou plus et une saturation en oxygène de $93 \%$ ou moins à l'air ambiant, ou des résultats évocateurs d'une pneumonie ${ }^{24}$. Quant à la COVID-19 légère, elle se caractérisait par un résultat positif au test de dépistage du SRAS-CoV-2 sans présence de symptômes graves. Nous avons classé les cas de COVID-19 comme étant symptomatiques ou asymptomatiques et graves ou légers. Nous avons inclus dans les cas de COVID-19 symptomatiques toutes les patientes présentant un symptôme, quelle qu'en soit la gravité. Pour les cas de COVID-19 grave, nous avons inclus les patientes présentant des symptômes graves et critiques, tandis que la COVID-19 légère incluait les cas asymptomatiques ou légers ${ }^{6,7,19}$.

\section{Issues}

La sélection des issues s'est faite en fonction de leur importance clinique et de l'accès aux données pertinentes dans les études publiées. Les issues primaires étaient la prééclampsie et la naissance prématurée. Les issues secondaires étaient les suivantes : diabète gestationnel, chorioamnionite ou infection intra-amniotique, césarienne, anomalies de la fonction hépatique, lymphopénie, ventilation mécanique, admission en unité de soins intensifs (USI), mortinaissance (mort fœtale à partir de la $20^{\mathrm{e}}$ semaine de grossesse), détresse fotale, poids à la naissance, faible poids à la naissance, âge gestationnel à la naissance, admission en unité de soins intensifs néonatals (USIN) et décès néonatal. 


\section{Évaluation de la qualité des études}

Deux examinatricess ont évalué de manière indépendante la qualité méthodologique de chaque étude selon l'échelle de Newcastle-Ottawa ${ }^{23}$. Les études de cohorte ont été évaluées selon les 3 critères suivants : qualité de la sélection des cohortes (4 étoiles), comparabilité des cohortes (2 étoiles) et évaluation des issues (3 étoiles). Les études cas-témoins ont été évaluées en fonction de la qualité de la sélection des cas et des témoins (4 étoiles), la comparabilité des cas et des témoins (2 étoiles) et la détermination de l'exposition (3 étoiles). Nous avons jugé qu'un total de 7 étoiles sur 9 correspondait à un faible risque de biais, que 4 à 6 étoiles correspondaient à un risque modéré et que moins de 4 étoiles correspondaient à un risque élevé de biais (annexe 2, accessible en anglais au www.cmaj.ca/lookup/ doi/10.1503/cmaj.202604/tab-related-content). En cas de désaccord, les examinatrices sont parvenues à un consensus lors d'une discussion avec un troisième examinateur.

\section{Extraction et analyse des données}

Deux auteurs ont extrait les données indépendamment et en double, toute divergence étant résolue par une discussion. Les données ont été extraites et l'analyse statistique a été effectuée dans Review Manager 5.4. Pour la méta-analyse, nous avons eu recours à des estimations non ajustées, car la plupart des études ne rapportaient des données que pour les issues brutes. Nous avons utilisé le test de Mantel-Haenszel pour combiner les données des variables dichotomiques, et les mesures de l'effet sont présentées sous forme de rapports de cotes (RC) avec des intervalles de confiance (IC) à $95 \%$. Pour les variables continues, nous avons calculé la différence moyenne (DM) pondérée en fonction de la taille de l'échantillon lorsque les issues étaient mesurées de la même manière d'une étude à l'autre. Nous avons évalué les associations entre la morbidité liée à la COVID-19 et les issues de grossesse (personnes atteintes de la COVID-19 par rapport aux personnes enceintes non infectées par le SRAS-CoV-2). Nous avons également évalué les issues de la COVID-19 en fonction de la gravité chez les patientes enceintes dont l'infection a été confirmée (cas symptomatiques par rapport aux asymptomatiques, cas graves par rapport aux cas légers).

Nous avons utilisé des graphiques en forêt pour montrer les estimations ponctuelles (IC à $95 \%$ ) pour chaque étude, et un losange pour représenter l'estimation ponctuelle groupée (IC à $95 \%)$ pour chaque issue à l'étude. Nous avons évalué l'hétérogénéité à l'aide de l'indicateur $R^{2}$. Si la valeur de $R^{2}$ était égale ou supérieure à $40 \%$, nous avons considéré qu'il y avait hétérogénéité ${ }^{25}$. Ensuite, nous avons regroupé les résultats à l'aide de modèles à effets aléatoires, puis nous avons effectué une analyse de sensibilité en excluant les prépublications et les études présentant un risque modéré de biais et utilisé des modèles à effet fixe pour les issues des études comptant un petit nombre de patientes. Le risque de biais de publication a été évalué à l'aide d'un diagramme en entonnoir.

\section{Approbation éthique}

Il n'a pas été nécessaire de demander l'approbation éthique pour cet examen systématique, car les données étaient accessibles au public.

\section{Résultats}

Nous avons recensé 7212 citations potentiellement pertinentes au moyen de notre stratégie de recherche. Le diagramme de l'approche PRISMA (figure 1 ) résume le processus de recherche documentaire et de sélection des études. Après examen des titres et des résumés, nous avons lu 357 articles dans leur intégralité. Quarante-deux études observationnelles ${ }^{7-15,17,19,21,22,26-54}$ portant sur 438548 personnes enceintes répondaient aux critères d'inclusion et ont été retenues pour la revue systématique et la méta-analyse. Notre évaluation de la qualité méthodologique de chaque étude admissible est résumée à l'annexe 2. Quatre-vingt-quinze pour cent des études observationnelles (40 sur 42) présentaient un faible risque global de biais selon l'échelle de Newcastle-Ottawa, et $5 \%$ ( 2 sur 42) des études présentaient un risque modéré.

Les caractéristiques des études incluses sont résumées à l'annexe 3, accessible en anglais au www.cmaj.ca/lookup/ doi/10.1503/cmaj.202604/tab-related-content. Sur les 42 études, 16 portaient sur des cohortes prospectives ${ }^{7,9,11,18,19,21,28,30,33,34,36,37,46,49-51}$, 21 , sur des cohortes rétrospectives ${ }^{8,10,12-14,17,27,29,32,35,38-45,48,53,54}$ et 5 , sur des études cas-témoins ${ }^{22,31,47,50,52}$. Une étude prospective a donné lieu à 2 publications, soit une étude en prépublication portant sur un résultat positif à l'infection par rapport à un résultat négatif au dépistage du SRAS-CoV- $2^{34}$, et une autre portant sur une infection grave comparée à une infection légère par la COVID-19 ${ }^{18}$. Les cas de COVID-19 ont été confirmés par un test de dépistage par PCR. Vingt-huit études portaient sur une infection par le SRASCoV-2 confirmée durant la grossesse comparativement à l'absence d'infection 11-13,15,22,27-32,34,35,37,39-45,47-51,53,54. Douze études ont mis en parallèle la COVID-19 symptomatique et asymptomatique $e^{7-15,46,48,52}$. Enfin, 13 études ont comparé la COVID-19 grave et légère pendant la grossesse $\mathrm{e}^{8,9,15,17-19,21,22,26,33,36,38,48}$.

Comparativement à l'absence d'infection, l'infection par le SRASCoV-2 pendant la grossesse était associée à la prééclampsie $(\mathrm{RC} 1,33$; IC à 95\% 1,03-1,73; $R=31 \%$; 13 études) (figure 2A), à l'accouchement prématuré (RC 1,82; IC à $95 \% 1,38-2,39 ; I^{2}=64 \%$; 18 études) (figure $3 A$ ), à la mortinaissance (RC 2,11; IC à $95 \% 1,14-3,90 ; I^{2}=24 \%$; 6 études) (figure 4), l'admission à l'USI (RC 4,78; IC à 95\% 2,03-11,25; $R^{2}=76 \% ; 5$ études), le faible poids à la naissance (grammes; différence moyenne : $-8,96$; IC à $95 \%-130,22$ à $-7,69 ; I^{2}=29 \%$; 13 études) et l'admission à l'USIN (RC 3,69; IC à 95\% 1,39-9,82; $R=$ $94 \%$; 10 études) (annexe 4, accessible en anglais au www.cmaj.ca/ lookup/doi/10.1503/cmaj.202604/tab-related-content). Comparativement à l'absence de maladie, il n'y avait pas d'association entre la COVID-19 et le diabète gestationnel, l'accouchement par césarienne, l'hémorragie post-partum et le décès néonatal (tableau 1).

Par rapport à la COVID-19 asymptomatique, la COVID-19 symptomatique pendant la grossesse a été associée à un risque accru de naissance prématurée (RC 2,29; IC à $95 \% 1,49-3,53 ; I^{2}=57 \%$; 9 études) (figure 3B) et d'accouchement par césarienne (RC 1,57; IC à $95 \% 1,32-1,85 ; P^{2}=1 \%$; 9 études) (annexe 4). La COVID-19 symptomatique n'était pas associée au diabète gestationnel (tableau 1).

Par rapport à la COVID-19 légère, la COVID-19 grave était fortement associée à la prééclampsie (RC 4,16; IC à $95 \%$ 1,5511,$15 ; I^{2}=0 \%$; 5 études) (figure $2 \mathrm{C}$ ), à la naissance prématurée (RC 4,29; IC à $95 \% 2,41-7,63 ; I^{2}=61 \%$; 10 études) (figure 3C), au 

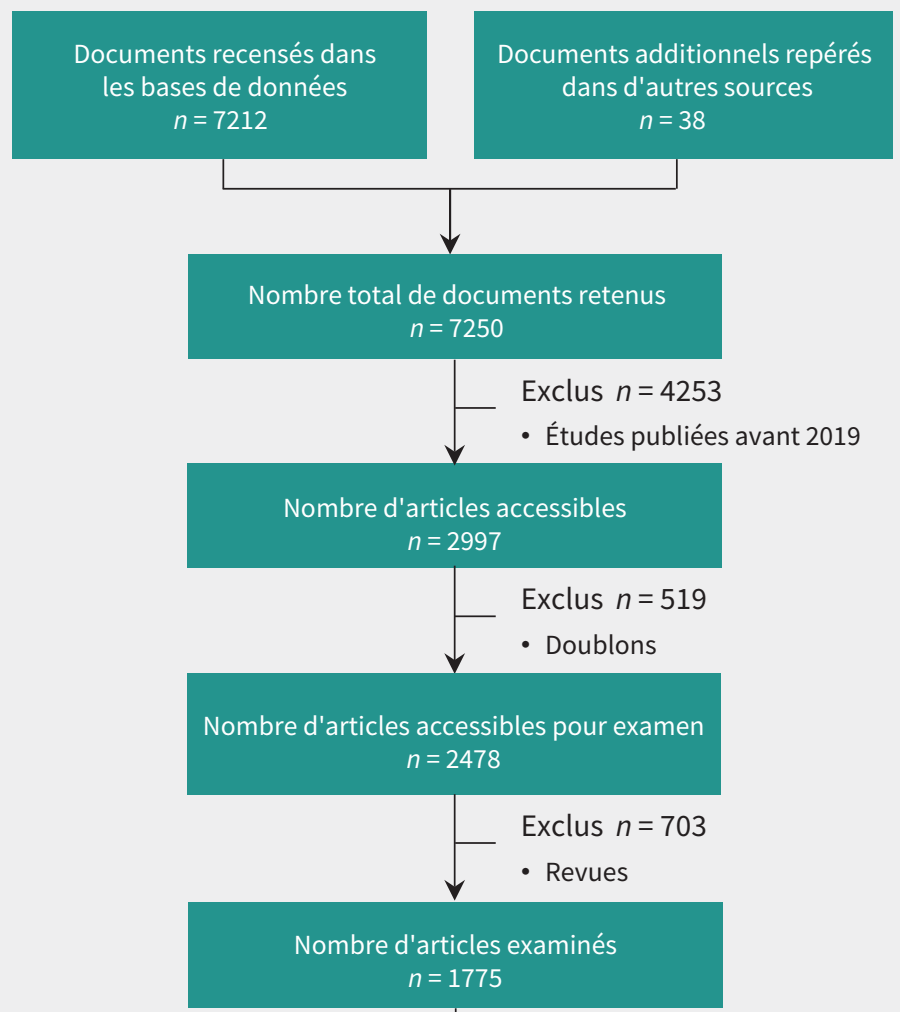

Exclus $n=1418$

- Jugés non pertinents après examen des titres et des résumés
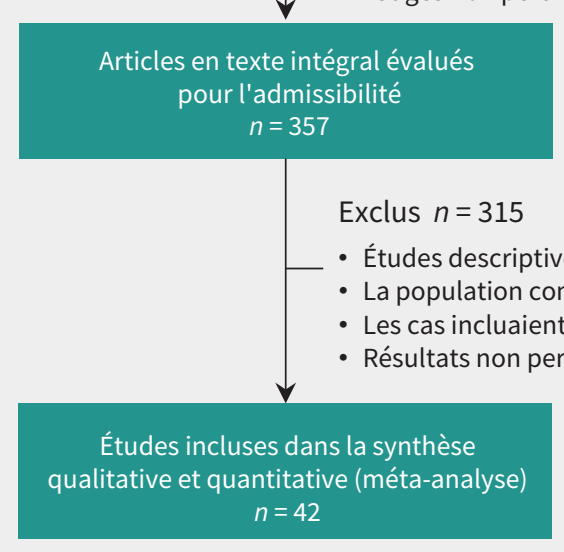

Figure 1 : Organigramme pour la sélection des études.

diabète gestationnel (RC 1,99; IC à $95 \% 1,09-3,64 ; I^{2}=14 \%$; 5 études), à l'admission à l'USI (RC 15,46; IC à 95\% 5,79-41,23; $P^{2}=0 \%$; 5 études), à la ventilation mécanique (RC 19,31; IC à $95 \%$ 9,38-39,72; $1^{2}=0 \%$; 5 études), à l'accouchement par césarienne ( $R C$ 2,58; IC à $95 \%$ 1,64-4,06; $1^{2}=43 \%$; 8 études), au faible poids à la naissance (RC 1,89; IC à $95 \% 1,14-3,12 ; I^{2}=0 \%$; 2 études) et à l'admission à l'USIN (RC 3,95; IC à 95\% 1,43-10,95; $r^{2}=79 \%$; 5 études) (tableau 1 et annexe 4).

Les analyses de sensibilité ont donné des résultats similaires (annexes 5 et 6, accessibles en anglais au www.cmaj.ca/lookup/ doi/10.1503/cmaj.202604/tab-related-content). Les diagrammes en entonnoir pour la prééclampsie et la naissance prématurée ne semblent pas indiquer que les conclusions étaient influencées par un biais de publication (annexe 7, accessible en anglais au www. cmaj.ca/lookup/doi/10.1503/cmaj.202604/tab-related-content).

\section{Interprétation}

Comparativement à l'absence de COVID-19 pendant la grossesse, nous avons constaté que la COVID-19 était associée à la prééclampsie, à la mortinaissance et à la naissance prématurée. Par rapport à la COVID-19 asymptomatique, la COVID-19 symptomatique était 
A

\begin{tabular}{|c|c|c|c|c|c|}
\hline \multirow[b]{2}{*}{ Étude ou sous-groupe } & \multicolumn{2}{|c|}{ COVID-19 } & \multicolumn{2}{|c|}{ Sans COVID-19 } & \multirow[b]{2}{*}{ RC (IC à $95 \%$ ) } \\
\hline & Événements & Total & Événements & Total & \\
\hline Adhikari et al. ${ }^{48}$ & 26 & 245 & 359 & 3035 & $0,88$ (0,58 à 1,35$)$ \\
\hline Ahlberg et al. ${ }^{43}$ & 12 & 155 & 26 & 604 & $1,87(0,92$ à 3,79$)$ \\
\hline Brandt et al. ${ }^{22}$ & 6 & 61 & 10 & 122 & 1,22 (0,42 à 3,53) \\
\hline Erol et al. ${ }^{50}$ & 2 & 60 & 0 & 36 & $3,12(0,15$ à 66,83$)$ \\
\hline Gulersen et al. ${ }^{29}$ & 5 & 50 & 7 & 50 & 0,68 (0,20 à 2,32) \\
\hline Jering et al..$^{53}$ & 564 & 6380 & 27078 & 400066 & 1,34 (1,22 à 1,46) \\
\hline Martínez-Perez et al. ${ }^{18}$ & 11 & 246 & 44 & 763 & $0,76$ (0,39 à 1,51$)$ \\
\hline Patberg et al. ${ }^{41}$ & 5 & 77 & 0 & 56 & $8,57(0,46$ à 158,29$)$ \\
\hline Pirjani et al. ${ }^{37}$ & 6 & 66 & 4 & 133 & $3,23(0,88$ à 11,85$)$ \\
\hline Wang et al. ${ }^{39}$ & 10 & 53 & 59 & 760 & 2,76 (1,32 à 5,78) \\
\hline Yang et al. ${ }^{42}$ & 1 & 65 & 83 & 11013 & $2,06(0,28$ à 15,01$)$ \\
\hline Yazihan et al. ${ }^{47}$ & 3 & 95 & 0 & 92 & $7,00$ (0,36 à 137,43$)$ \\
\hline Zhang et al. ${ }^{40}$ & 1 & 16 & 4 & 45 & 0,68 (0,07 à 6,61) \\
\hline Total (IC à 95 \%) & & 7569 & & 416775 & 1,33 (1,03 à 1,73) \\
\hline $\begin{array}{l}\text { Total des événements } \\
\text { Hétérogénéité : } I^{2}=31 \%\end{array}$ & 652 & & 27674 & & \\
\hline
\end{tabular}

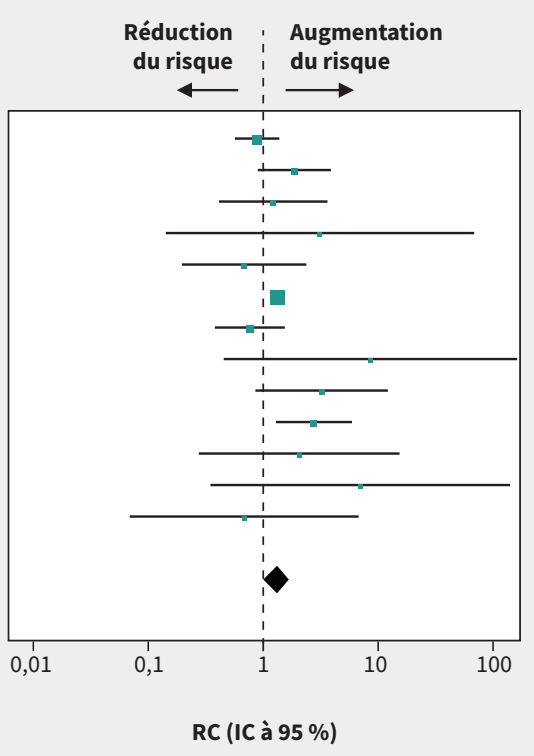

B

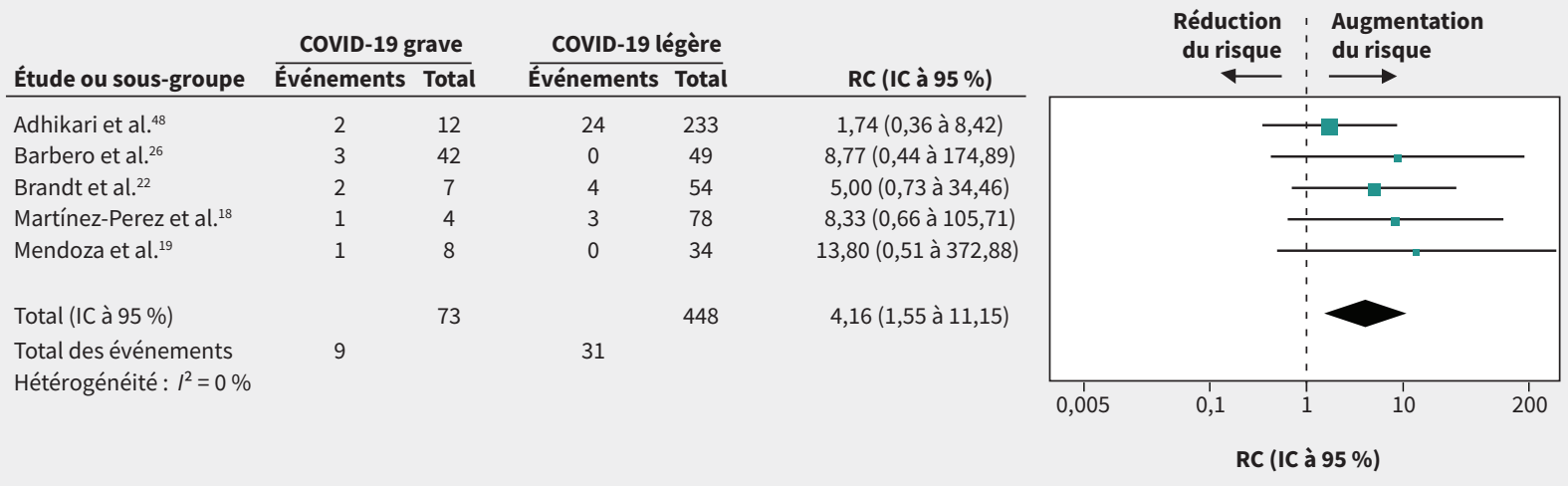

Figure 2 : Graphiques en forêt des rapports de cotes (RC) bruts sommaires avec intervalles de confiance (IC) à 95\% pour l'association entre la maladie à coronavirus 2019 (COVID-19) et la prééclampsie. A) Association entre COVID-19 et prééclampsie (patientes avec COVID-19 comparativement aux patientes sans COVID-19). B) Association entre COVID-19 grave et prééclampsie (patientes avec COVID-19 grave comparativement aux patientes avec COVID-19 légère).

associée à un risque accru de césarienne et de naissance prématurée. Et la COVID-19 grave était fortement associée à la prééclampsie, au diabète gestationnel, à la naissance prématurée et au faible poids à la naissance, par rapport à la COVID-19 légère. Notre métaanalyse d'études observationnelles a le mérite unique de fournir des données comparatives sur la morbidité associée à la COVID-19 durant la grossesse. D'après nos observations, le fait de contracter la COVID-19 pendant la grossesse est associé à la prééclampsie et à la naissance prématurée, et la COVID-19 grave peut entraîner une morbidité maternelle et néonatale considérable.

Nous avons retenu des études présentant un risque de biais faible à modéré, en utilisant des critères stricts d'évaluation de la qualité23. Bien que le nombre de publications sur la COVID-19 chez les personnes enceintes ne cesse d'augmenter, les revues systématiques précédentes concernant la COVID-19 pendant la grossesse avaient principalement inclus des études de cas et des séries de $\operatorname{cas}^{4,5}$, ou présentaient une revue d'études de cas pour d'autres types de coronavirus ${ }^{55}$, décrivant la proportion de patientes présentant des manifestations cliniques ou des complications de la grossesse. Les auteurs de ces revues étaient conscients du manque de données de bonne qualité au début de la pandémie, données qui étaient nécessaires pour tirer des conclusions non biaisées. L'une des premières revues systématiques sur le sujet n'a observé aucune différence dans les caractéristiques cliniques des patientes atteintes de la COVID-19 qui étaient enceintes par rapport à celles qui n'étaient pas enceintes ${ }^{56}$. Une revue systématique en continu portant principalement sur des études de cas et des séries de $\operatorname{cas}^{5}$, dont beaucoup utilisaient des groupes témoins sans personnes enceintes, ou n'avaient pas de groupe témoin, a rapporté que les patientes enceintes étaient moins susceptibles de présenter des symptômes de la COVID-19 que les personnes atteintes de la COVID-19 qui n'étaient pas enceintes ${ }^{5}$. Notre méta-analyse d'études de cohorte récentes de bonne qualité comportant des données 


\begin{tabular}{|c|c|c|c|c|c|}
\hline \multirow[b]{2}{*}{ Étude ou sous-groupe } & \multicolumn{2}{|c|}{ COVID-19 } & \multicolumn{2}{|c|}{ Sans COVID-19 } & \multirow[b]{2}{*}{ RC (IC à $95 \%)$} \\
\hline & Événements & Total & Événements & Total & \\
\hline Ahlberg et al. ${ }^{43}$ & 14 & 155 & 45 & 604 & 1,23 (0,66 à 2,31) \\
\hline Cunarro-Lopez et al. ${ }^{44}$ & 13 & 68 & 2 & 43 & $4,85$ (1,04 à 22,66$)$ \\
\hline Diaz-Corvillon et al. ${ }^{15}$ & 4 & 37 & 27 & 549 & $2,34(0,77$ à 7,09$)$ \\
\hline Edlow et al. ${ }^{49}$ & 10 & 64 & 5 & 63 & 2,15 (0,69 à 6,69) \\
\hline Flaherman et al..$^{27}$ & 21 & 179 & 9 & 84 & $1,11(0,48$ à 2,53$)$ \\
\hline Hcini et al. ${ }^{51}$ & 11 & 137 & 36 & 370 & $0,81(0,40$ à 1,64$)$ \\
\hline Jering et al. ${ }^{53}$ & 459 & 6380 & 23234 & 400066 & $1,26(1,14$ à 1,38$)$ \\
\hline Li et al. ${ }^{31}$ & 3 & 16 & 6 & 121 & $4,42(0,99$ à 19,82$)$ \\
\hline Martínez-Perez et al. ${ }^{18}$ & 34 & 246 & 51 & 763 & 2,24 (1,41 à 3,55) \\
\hline Nayak et al. ${ }^{35}$ & 38 & 141 & 90 & 836 & 3,06 (1,99 à 4,71) \\
\hline Pineles et al. ${ }^{54}$ & 5 & 77 & 90 & 858 & $0,59(0,23$ à 1,51$)$ \\
\hline Prabhu et al. ${ }^{11}$ & 11 & 70 & 57 & 605 & $1,79(0,89$ à 3,61$)$ \\
\hline Smithgall et al. ${ }^{13}$ & 10 & 51 & 4 & 25 & $1,28(0,36$ à 4,57$)$ \\
\hline Wang et al. ${ }^{39}$ & 9 & 53 & 66 & 760 & 2,15 (1,01 à 4,60) \\
\hline Woodworth et al. ${ }^{46}$ & 8 & 16 & 99 & 594 & $5,00(1,83$ à 13,64$)$ \\
\hline Yang et al. ${ }^{42}$ & 9 & 65 & 579 & 11013 & $2,90(1,43$ à 5,88$)$ \\
\hline Yazihan et al. ${ }^{47}$ & 3 & 95 & 0 & 92 & $7,00(0,36$ à 137,43$)$ \\
\hline Zhang et al. ${ }^{40}$ & 3 & 16 & 6 & 45 & $1,50(0,33$ à 6,87$)$ \\
\hline Total (IC à $95 \%)$ & & 7866 & & 417491 & $1,82$ (1,38 à 2,39$)$ \\
\hline Total des événements & 665 & & 24406 & & \\
\hline
\end{tabular}

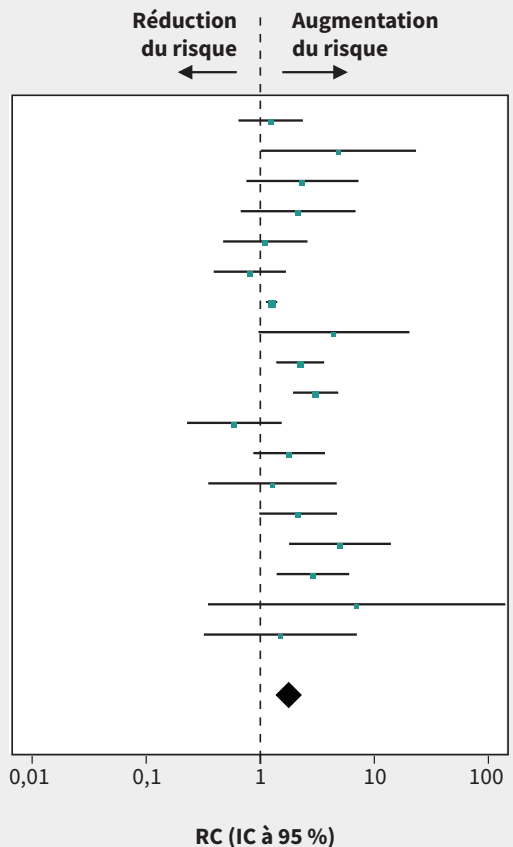

B

\begin{tabular}{|c|c|c|c|c|c|}
\hline \multirow[b]{2}{*}{ Étude ou sous-groupe } & \multicolumn{2}{|c|}{$\begin{array}{c}\text { COVID-19 } \\
\text { symptomatique }\end{array}$} & \multicolumn{2}{|c|}{$\begin{array}{c}\text { COVID-19 } \\
\text { asymptomatique }\end{array}$} & \multirow[b]{2}{*}{ RC (IC à $95 \%)$} \\
\hline & Événements & $\overline{\text { Total }}$ & Événements & Total & \\
\hline Adhikari et al. ${ }^{48}$ & 20 & 147 & 7 & 98 & 2,05 (0,83 à 5,04) \\
\hline Delahoy et al. ${ }^{7}$ & 31 & 134 & 25 & 311 & 3,44 (1,94 à 6,11) \\
\hline Di Mascio et al. ${ }^{55}$ & 60 & 189 & 10 & 77 & 3,12 (1,50 à 6,48) \\
\hline Jenabi et al..$^{52}$ & 12 & 45 & 6 & 45 & 2,36 (0,80 à 6,99) \\
\hline Khoury et al. ${ }^{9}$ & 25 & 139 & 11 & 102 & 1,81 (0,85 à 3,88) \\
\hline London et al. ${ }^{10}$ & 9 & 33 & 0 & 22 & 17,45 (0,96 à 317,38) \\
\hline Smithgall et al. ${ }^{13}$ & 6 & 25 & 4 & 26 & $1,74(0,43$ à 7,09$)$ \\
\hline Verma et al. ${ }^{14}$ & 14 & 89 & 2 & 60 & $5,41$ (1,18 à 24,77$)$ \\
\hline Woodworth et al. ${ }^{46}$ & 297 & 2315 & 43 & 376 & $1,14(0,81$ à 1,60$)$ \\
\hline Total (IC à $95 \%$ ) & & 3116 & & 1117 & 2,29 (1,49 à 3,53) \\
\hline Total des événements & 474 & & 108 & & \\
\hline
\end{tabular}

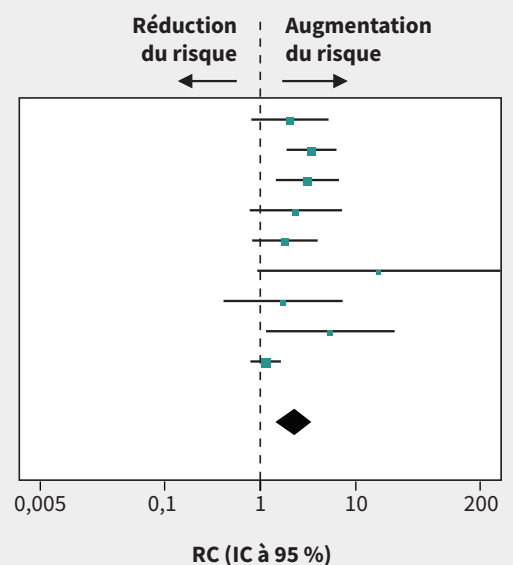

C

\begin{tabular}{|c|c|c|c|c|c|}
\hline \multirow[b]{2}{*}{ Étude ou sous-groupe } & \multicolumn{2}{|c|}{ COVID-19 grave } & \multicolumn{2}{|c|}{ COVID-19 légère } & \multirow[b]{2}{*}{ RC (IC à $95 \%)$} \\
\hline & Événements & Total & Événements & Total & \\
\hline Adhikari et al. ${ }^{48}$ & 5 & 12 & 22 & 233 & 6,85 (2,00 à 23,41) \\
\hline Barbero et al. ${ }^{26}$ & 5 & 11 & 3 & 12 & $2,50$ (0,43 à 14,61$)$ \\
\hline Brandt et al..$^{22}$ & 4 & 7 & 3 & 54 & $22,67$ (3,40 à 151,02$)$ \\
\hline Di Mascio et al. ${ }^{55}$ & 60 & 189 & 10 & 77 & $3,12(1,50$ à 6,48$)$ \\
\hline Kayem et al. ${ }^{17}$ & 37 & 58 & 13 & 123 & $14,91$ (6,80 à 32,70$)$ \\
\hline Khoury et al. ${ }^{9}$ & 18 & 73 & 18 & 166 & 2,69 (1,31 à 5,54) \\
\hline Maraschini et al. ${ }^{33}$ & 15 & 47 & 13 & 99 & 3,10 (1,33 à 7,23) \\
\hline Martínez-Perez et al. ${ }^{18}$ & 4 & 4 & 21 & 78 & $24,07(1,24$ à 466,10$)$ \\
\hline Panagiotakopoulos et al. ${ }^{36}$ & 5 & 32 & 9 & 61 & 1,07 (0,33 à 3,51) \\
\hline Savasi et al. ${ }^{21}$ & 4 & 11 & 8 & 46 & 2,71 (0,64 à 11,52) \\
\hline Total (IC à $95 \%$ ) & & 444 & & 949 & 4,29 (2,41 à 7,63) \\
\hline $\begin{array}{l}\text { Total des événements } \\
\text { Hétérogénéité: } I^{2}=61 \%\end{array}$ & 157 & & 120 & & \\
\hline
\end{tabular}

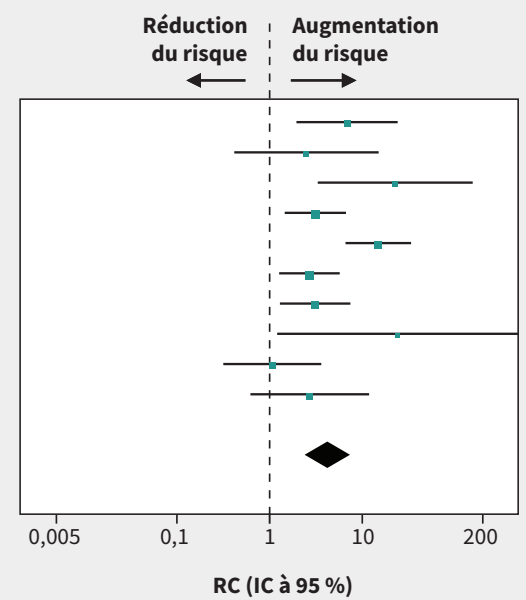

Figure 3 : Graphiques en forêt des rapports de cotes (RC) bruts sommaires avec intervalles de confiance (IC) à $95 \%$ pour l'association entre la maladie à coronavirus 2019 (COVID-19) et la naissance prématurée. A) Association entre COVID-19 et naissance prématurée (patientes avec COVID-19 comparativement aux patientes sans COVID-19). B) Association entre COVID-19 symptomatique et naissance prématurée (patientes avec COVID-19 symptomatique comparativement aux patientes avec COVID-19 asymptomatique). C) Association entre COVID-19 grave et naissance prématurée (patientes avec COVID-19 grave comparativement aux patientes avec COVID-19 légère). 


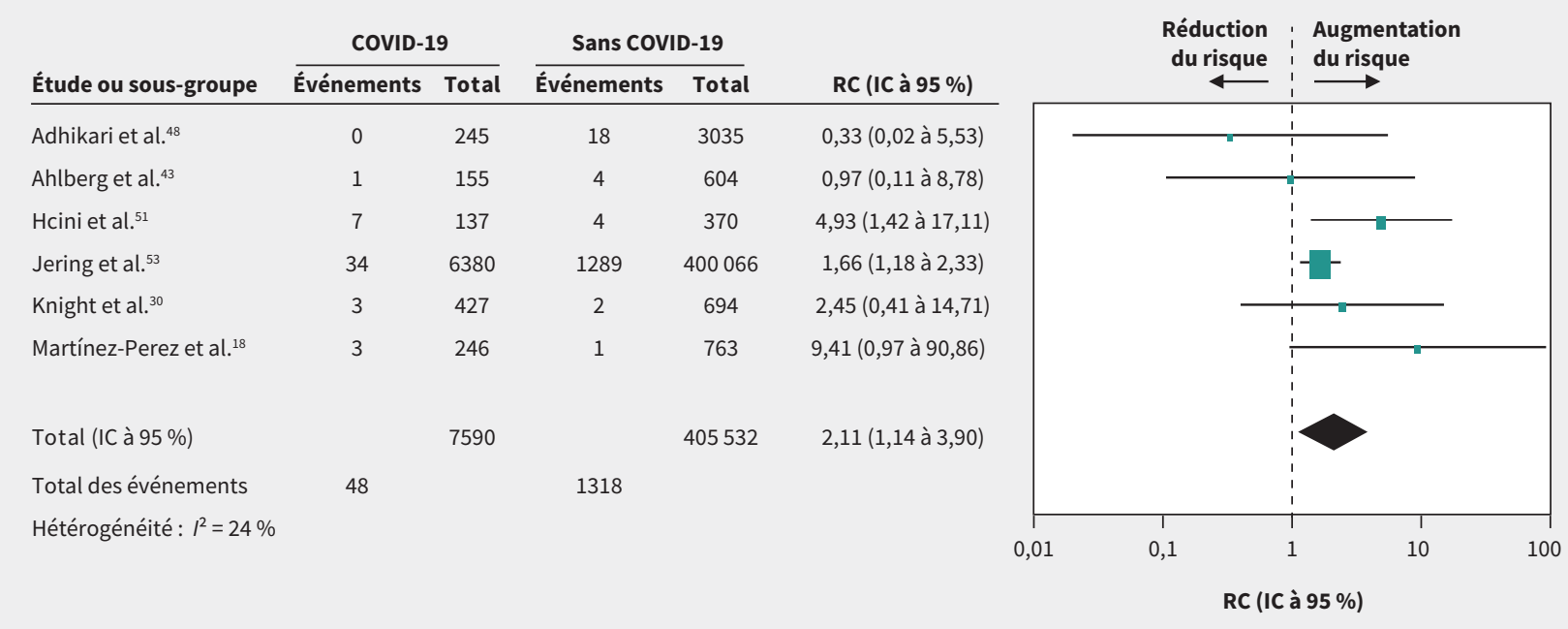

Figure 4 : Graphique en forêt des rapports de cotes (RC) bruts résumés avec intervalles de confiance (IC) à $95 \%$ pour l'association entre la maladie à coronavirus 2019 (COVID-19) et la mortinaissance.

comparatives arrive à une conclusion différente, et fournit des preuves tangibles que la COVID-19 symptomatique ou grave est associée à un risque considérable de prééclampsie, de naissance prématurée et de faible poids à la naissance.

Les mécanismes qui sous-tendent l'association entre la COVID-19 et la prééclampsie ne sont pas bien définis, mais des chercheurs ont montré que le SRAS-CoV-2 entraîne un dysfonctionnement du système rénine-angiotensine et une vasoconstriction en se liant au récepteur de l'enzyme de conversion de l'angiotensine $2^{57}$. La prééclampsie, qui se caractérise par un dysfonctionnement endothélial systémique, pourrait avoir un mécanisme en commun avec la COVID-19, les effets vasculaires de l'infection par le SRAS-CoV-2 étant de plus en plus reconnus. Une étude a révélé que les personnes enceintes atteintes d'une forme grave de COVID-19 présentaient des manifestations cliniques comparables à la prééclampsie, qui s'en distinguait par des taux de biomarqueurs, notamment la tyrosine kinase analogue à FMS soluble dans le sérum et le facteur de croissance placentaire $^{19}$. Certaines études ont montré que l'infection par le SRAS-CoV-2 créait un état pro-inflammatoire suivi par un dysfonctionnement endothélial systémique et une prééclampsie ${ }^{58,59}$. Cette constatation concorde avec les conclusions d'une étude suédoise de 2020, qui avait rapporté une prévalence accrue de prééclampsie chez les personnes enceintes atteintes de la COVID-1943.

Notre méta-analyse semble également indiquer que comparativement à l'absence d'infection, l'infection par le SRAS-CoV-2 serait associée à la naissance prématurée, à la mortinaissance et au faible poids à la naissance, mais pas à l'accouchement par césarienne. Nous avons également observé que la forme grave de la COVID-19 était fortement associée à la naissance prématurée et à d'autres issues périnatales défavorables. Il se peut que certains de ces risques excessifs soient liés à la prééclampsie, même si l'infection par le SRAS-CoV-2 peut également provoquer la réponse inflammatoire systémique exagérée présente durant la pathogenèse d'une naissance prématurée ou être responsable d'un milieu sous-optimal pour la croissance et le développement du fœtus. Les résultats de l'analyse histopathologique du placenta de patientes atteintes de la COVID-19 au moment de l'accouchement ont révélé une malperfusion vasculaire placentaire ${ }^{41}$, un élément susceptible d'avoir un effet sur la croissance du fœtus, la mortinaissance et la naissance prématurée. Une récente étude quasi expérimentale nationale menée aux Pays-Bas a révélé une association entre les mesures d'atténuation de la COVID-19 et une réduction de l'incidence des naissances prématurées ${ }^{60}$.

L'absence d'information sur les effets de l'infection par le SRAS-CoV-2 lors de la grossesse suscite d'importants questionnements chez les obstétriciens et les néonatologistes, qui s'inquiètent du risque de morbidité et de mortalité maternelles, fœtales et néonatales. Il existe donc un besoin urgent de données probantes pour orienter les décisions cliniques. Les résultats que nous avons recueillis semblent indiquer que l'infection par le SRAS-CoV-2 augmenterait le risque de prééclampsie, de mortinaissance, de naissance prématurée et d'admission à I'USIN, et que le risque d'issues maternelles, fœtales et néonatales indésirables est particulièrement important chez les personnes enceintes atteintes d'une forme grave de la COVID-19. Les cliniciens doivent être informés de ces issues indésirables lorsqu'ils prennent en charge la grossesse de patientes atteintes de la COVID-19 afin d'adopter des stratégies efficaces pour prévenir ou réduire les risques pour les patientes et les fœtus.

\section{Limites}

Nous n'avons pas enregistré notre étude auprès du registre de l'International Prospective Register of Systematic Reviews (PROSPERO), et notre recherche documentaire était limitée aux publications en anglais. Malgré le grand nombre d'issues prises en compte, nous ne pouvons pas exclure la possibilité que certaines associations soient non fondées. Certaines des études retenues $(14 \%)$ n'exigeaient pas un résultat négatif à un test de 
Tableau 1 : Résumé des résultats regroupés au moyen de modèles à effets aléatoires

\begin{tabular}{|c|c|c|c|c|c|}
\hline Issues & $\begin{array}{c}\mathbf{N}^{\text {bre }} \\
\text { d'études }\end{array}$ & $\begin{array}{c}\mathrm{N}^{\text {bre }} \text { de } \\
\text { participants }\end{array}$ & $\begin{array}{c}\mathbf{N}^{\text {bre }} \\
\text { d'événements }\end{array}$ & $\begin{array}{l}\text { Différence moyenne } \\
\text { (IC à 95\%) }\end{array}$ & $\begin{array}{c}\text { RC } \\
\text { (IC à } 95 \%)\end{array}$ \\
\hline \multicolumn{6}{|l|}{ Patientes avec c. sans COVID-19 } \\
\hline Prééclampsie & 13 & 424344 & 28326 & & $1,33$ (1,03 à 1,73$)$ \\
\hline Diabète gestationnel & 13 & 425890 & 40567 & & $1,03(0,76$ à 1,39$)$ \\
\hline Détresse fœtale & 3 & 874 & 47 & & $1,50(0,64$ à 3,53$)$ \\
\hline Mortinaissance & 6 & 413122 & 1366 & & 2,11 (1,14 à 3,90) \\
\hline $\begin{array}{l}\text { Chorioamniotite ou } \\
\text { infection intra-amniotique }\end{array}$ & 5 & 4368 & 433 & & $0,85(0,57$ à 1,26$)$ \\
\hline Admission à l'USI & 5 & 409737 & 2012 & & $4,78$ (2,03 à 11,25$)$ \\
\hline Accouchement par césarienne & 22 & 429366 & 121650 & & $1,00(0,82$ à 1,23$)$ \\
\hline Hémorragie post-partum & 5 & 2981 & 355 & & $0,89(0,52$ à 1,53$)$ \\
\hline Naissance prématurée & 18 & 425357 & 25071 & & $1,82(1,38$ à 2,39$)$ \\
\hline Sexe néonatal, masculin & 5 & 11985 & 6369 & & $0,97(0,71$ à 1,33$)$ \\
\hline $\begin{array}{l}\text { Âge gestationnel à la } \\
\text { naissance, en semaines }\end{array}$ & 13 & 4197 & & $\begin{array}{c}-0,24 \\
(-0,49 \text { à } 0,00)\end{array}$ & \\
\hline $\begin{array}{l}\text { Poids à la naissance, } \\
\text { grammes }\end{array}$ & 13 & 2973 & & $\begin{array}{c}-68,96 \\
(-130,22 \text { à }-7,69)\end{array}$ & \\
\hline Faible poids à la naissance & 2 & 1054 & 678 & & $2,32(0,26$ à 21,07$)$ \\
\hline Admission à l'USIN & 10 & 5675 & 785 & & 3,69 (1,39 à 9,82) \\
\hline Décès néonatal & 5 & 2838 & & & $1,10(0,41$ à 2,95$)$ \\
\hline
\end{tabular}

$\%$

31

54

76

78

64

61

29

85

94

Patientes présentant une COVID-19 symptomatique c. asymptomatique

Hypertension gestationnelle
ou prééclampsie
Diabète gestationnel
Ventilation mécanique
Admission à l'USI
Accouchement par césarienne
Naissance prématurée
Admission à l'USIN
Décès néonatal

\begin{tabular}{cc}
4122 & 333 \\
\hline 3767 & 256 \\
1023 & 62 \\
1178 & 97 \\
4232 & 1406 \\
\hline 4233 & 582 \\
\hline 2365 & 248 \\
\hline 938 & 11 \\
\hline
\end{tabular}

$1,12(0,82$ à 1,55$)$

$16,29(3,88$ à 68,47$) \quad 0$

$7,40(0,48$ à 114,24$)$

$1,57(1,32$ à 1,85$) \quad 1$

$2,29(1,49$ à 3,53$)$

$3,47(0,38$ à 31,49$)$

$3,67(0,88$ à 15,29$)$

Patientes présentant une COVID-19 grave

c. légère

\begin{tabular}{lccc} 
Prééclampsie & 5 & 521 & 40 \\
Diabète gestationnel & 5 & 1140 & 105 \\
$\begin{array}{l}\text { Anomalie de la fonction } \\
\text { hépatique }\end{array}$ & 4 & 350 & 116 \\
$\begin{array}{l}\text { Lymphopénie } \\
\text { Admission à l'USI }\end{array}$ & 4 & 561 & 221 \\
\hline $\begin{array}{l}\text { Ventilation mécanique } \\
\text { Accouchement par césarienne }\end{array}$ & 5 & 757 & 70 \\
\hline Naissance prématurée & 10 & 962 & 97 \\
\hline $\begin{array}{l}\text { Âge gestationnel à la } \\
\text { naissance, en semaines }\end{array}$ & 8 & 1138 & 452 \\
\hline $\begin{array}{l}\text { Faible poids à la naissance } \\
\text { Admission à l'USIN }\end{array}$ & 2 & 709 & 277 \\
\hline Décès néonatal & 5 & 729 & 74 \\
& 3 & 827 & 12
\end{tabular}

4,16 (1,55 à 11,15$)$

$1,99(1,09$ à 3,64) $\quad 14$

$6,47(2,60$ à 16,09$)$

$3,04(1,93$ à 4,79$)$

$15,46(5,79$ à 41,23) $\quad 0$

$19,31(9,38$ à 39,72$)$

2,58 $(1,64$ à 4,06)

$4,29(2,41$ à 7,63$) \quad 61$

$-3,50$

91

$(-5,96$ à $-1,03)$

$\begin{array}{cc}1,89(1,14 \text { à } 3,12) & 0 \\ 3,95(1,43 \text { à } 10,95) & 79 \\ 33,71(5,18 \text { à } 219,44) & 0\end{array}$

Remarque : COVID-19 = maladie à coronavirus 2019, IC = intervalle de confiance, RC = rapport de cotes, USI = unité de soins intensifs, USIN = unité de soins intensifs néonatals 
dépistage par PCR pour l'inclusion dans le groupe témoin non exposé22,32,37,40,42,47. La taille des effets non ajustée a été estimée, ce qui pourrait avoir entraîné une surestimation des risques. Il ressort de l'étude la plus importante disposant de données sur les facteurs confusionnels que les associations entre la COVID-19 et les issues de grossesse étaient quelque peu atténuées après ajustement, même si les risques sont restés élevés pour la plupart des issues ${ }^{53}$. Par ailleurs, nous n'avons pas pu déterminer l'importance clinique de certaines des issues. À titre d'exemple, nous ne pouvons pas confirmer que tous les patients admis à I'USIN ont eu besoin de soins intensifs. Le motif de la naissance prématurée n'était pas clair : il n'a entre autres pas été possible de déterminer si la naissance prématurée était indiquée médicalement ou spontanée. Comme les données se fondaient sur l'observation, nous ne pouvons pas éliminer la possibilité d'un facteur de confusion résiduel.

\section{Conclusion}

L'infection par le SRAS-CoV-2 pendant la grossesse a été associée à des risques de prééclampsie, de mortinaissance, de naissance prématurée et d'admission à l'USIN. En outre, il y a une forte association entre l'infection par le SRAS-CoV-2 entraînant une maladie grave et la prééclampsie et d'autres issues défavorables, maternelles et néonatales. Il faudra mener d'autres études pour recueillir des données plus fiables afin de valider ou d'étayer ces résultats, de mieux connaître les mécanismes physiopathologiques qui expliquent ces associations et pour mettre en évidence des stratégies efficaces de prévention des issues défavorables chez les personnes enceintes atteintes de la COVID-19.

\section{Références}

1. Wang C, Horby PW, Hayden FG, et al. A novel coronavirus outbreak of global health concern. Lancet 2020;395:470-3.

2. Wastnedge EAN, Reynolds RM, van Boeckel SR, et al. Pregnancy and COVID-19. Physiol Rev 2021;101:303-18.

3. Dashraath P, Wong JLJ, Lim MXK, et al. Coronavirus disease 2019 (COVID-19) pandemic and pregnancy. Am J Obstet Gynecol 2020;222:521-31.

4. Huntley BJF, Huntley ES, Di Mascio D, et al. Rates of maternal and perinatal mortality and vertical transmission in pregnancies complicated by severe acute respiratory syndrome coronavirus 2 (SARS-CoV-2) infection: a systematic review. Obstet Gynecol 2020;136:303-12.

5. Allotey J, Stallings E, Bonet M, et al. Clinical manifestations, risk factors, and maternal and perinatal outcomes of coronavirus disease 2019 in pregnancy: living systematic review and meta-analysis. BMJ 2020;370:m3320.

6. Breslin N, Baptiste C, Gyamfi-Bannerman C, et al. COVID-19 infection among asymptomatic and symptomatic pregnant women: two weeks of confirmed presentations to an affiliated pair of New York City hospitals. Am J Obstet Gynecol MFM 2020;2:100118.

7. Delahoy MJ, Whitaker M, O'Halloran A, et al. Characteristics and maternal and birth outcomes of hospitalized pregnant women with laboratory-confirmed COVID-19 - COVID-NET, 13 states, March 1-August 22, 2020. MMWR Morb Mortal Wkly Rep 2020;69:1347-54.

8. WAPM (World Association of Perinatal Medicine) Working Group on COVID-19. Maternal and perinatal outcomes of pregnant women with SARS-CoV-2 infection. Ultrasound Obstet Gynecol 2021;57:232-41.

9. Khoury R, Bernstein PS, Debolt C, et al. Characteristics and outcomes of 241 births to women with severe acute respiratory syndrome coronavirus 2 (SARSCoV-2) infection at five New York City medical centers. Obstet Gynecol 2020;136:273-82.

10. London V, McLaren R, Atallah F, et al. The relationship between status at presentation and outcomes among pregnant women with COVID-19. Am J Perinatol 2020;37:991-4.
11. Prabhu M, Cagino K, Matthews KC, et al. Pregnancy and postpartum outcomes in a universally tested population for SARS-CoV-2 in New York City: a prospective cohort study. BJOG 2020;127:1548-56.

12. Sakowicz A, Ayala AE, Ukeje CC, et al. Risk factors for severe acute respiratory syndrome coronavirus 2 infection in pregnant women. Am J Obstet Gynecol MFM 2020;2:100198.

13. Smithgall MC, Liu-Jarin X, Hamele-Bena D, et al. Third-trimester placentas of severe acute respiratory syndrome coronavirus 2 (SARS-CoV-2)-positive women: histomorphology, including viral immunohistochemistry and in-situ hybridization. Histopathology 2020;77:994-9.

14. Verma S, Bradshaw C, Auyeung NSF, et al. Outcomes of maternal-newborn dyads after maternal SARS-CoV-2. Pediatrics 2020;146:e2020005637.

15. Díaz-Corvillón P, Mönckeberg M, Barros A, et al. Routine screening for SARSCoV-2 in unselected pregnant women at delivery. PLoS One 2020;15:e0239887.

16. Cohen J, Vignaux O, Jacquemard F. Covid-19 in pregnant women: general data from a French National Survey. Eur J Obstet Gynecol Reprod Biol 2020;251:267-8.

17. Kayem G, Lecarpentier E, Deruelle P, et al. A snapshot of the COVID-19 pandemic among pregnant women in France. J Gynecol Obstet Hum Reprod 2020;49:101826.

18. Martínez-Perez O, Vouga M, Cruz Melguizo S, et al. Association between mode of delivery among pregnant women with COVID-19 and maternal and neonatal outcomes in Spain. JAMA 2020;324:296-9.

19. Mendoza M, Garcia-Ruiz I, Maiz N, et al. Preeclampsia-like syndrome induced by severe COVID-19: a prospective observational study. BJOG 2020;127:1374-80.

20. Onwuzurike $C$, Diouf $\mathrm{K}$, Meadows AR, et al. Racial and ethnic disparities in severity of COVID-19 disease in pregnancy in the United States. Int J Gynaecol Obstet 2020;151:293-5.

21. Savasi VM, Parisi F, Patanè L, et al. Clinical findings and disease severity in hospitalized pregnant women with coronavirus disease 2019 (COVID-19). Obstet Gynecol 2020;136:252-8.

22. Brandt JS, Hill J, Reddy A, et al. Epidemiology of coronavirus disease 2019 in pregnancy: risk factors and associations with adverse maternal and neonatal outcomes. Am J Obstet Gynecol 2020;S0002-9378:31134-0.

23. Wells G, Shea B, O'connell D, et al. The Newcastle-Ottawa Scale (NOS) for Assessing the Quality of Nonrandomised Studies in Meta-Analyses. Ottawa: The Ottawa Hospital Research Institute; 2013. Accessible ici : www.ohri.ca/programs /clinical_epidemiology/oxford.asp (consulté le 10 sept. 2020).

24. Wu Z, McGoogan JM. Characteristics of and important lessons from the coronavirus disease 2019 (COVID-19) outbreak in China: summary of a report of 72314 cases from the Chinese Center for Disease Control and Prevention. JAMA 2020;323:1239-42.

25. Deeks JJ, Higgins JPT, Altman DG. Chapter 10: Analysing data and undertaking meta-analyses. In: Higgins JPT, Thomas J, Chandler J, et al., editors. Cochrane Handbook for Systematic Reviews of Interventions Version 6.1. Cochrane; 2020. Accessible ici : www.training.cochrane.org/handbook (consulté le 22 janv. 2021).

26. Barbero P, Mugüerza L, Herraiz I, et al. SARS-CoV-2 in pregnancy: characteristics and outcomes of hospitalized and non-hospitalized women due to COVID-19. J Matern Fetal Neonatal Med 2020 Jul 20 [cyberpublication avant impression];1-7. doi: 10.1080/14767058.2020.1793320.

27. Flaherman VJ, Afshar Y, Boscardin J, et al. Infant outcomes following maternal infection with SARS-CoV-2: first report from the PRIORITY study. Clin Infect Dis 2020 Sept. 18 [cyberpublication avant impression]; ciaa1411. doi: 10.1093/cid/ciaa1411.

28. Griffin I, Benarba F, Peters C, et al. The impact of COVID-19 infection on labor and delivery, newborn nursery, and neonatal intensive care unit: prospective observational data from a single hospital system. Am J Perinatol 2020;37:1022-30.

29. Gulersen M, Prasannan L, Tam Tam H, et al. Histopathologic evaluation of placentas after diagnosis of maternal severe acute respiratory syndrome coronavirus 2 infection. Am J Obstet Gynecol MFM 2020;2:100211.

30. Knight M, Bunch K, Vousden N, et al. Characteristics and outcomes of pregnant women admitted to hospital with confirmed SARS-CoV-2 infection in UK: national population-based cohort study. BMJ 2020;369:m2107.

31. Li N, Han L, Peng M, et al. Maternal and neonatal outcomes of pregnant women with COVID-19 pneumonia: a case-control study. Clin Infect Dis 2020;71:2035-41.

32. Liu Y, Chen H, Tang K, et al. Clinical manifestations and outcome of SARSCoV-2 infection during pregnancy. J Infect 2020 Mar. 4 [cyberpublication avant impression]. doi: 10.1016/j.jinf.2020.02.028.

33. Maraschini A, Corsi E, Salvatore MA, et al. Coronavirus and birth in Italy: results of a national population-based cohort study. Ann Ist Super Sanita 2020;56:378-89.

34. Martinez-Perez O, Rodriguez PP, Hernandez MM, et al. The association between COVID-19 and preterm delivery: a cohort study with a multivariate analysis [préimpression]. medRxiv 2020 Sept. 7. Accessible ici : https://doi.org/10.1101 /2020.09.05.20188458 (consulté le 20 janv. 2021). 
35. Nayak AH, Kapote DS, Fonseca M, et al. Impact of the coronavirus infection in pregnancy: a preliminary study of 141 patients. J Obstet Gynaecol India 2020;70:256-61.

36. Panagiotakopoulos L, Myers TR, Gee J, et al. SARS-CoV-2 infection among hospitalized pregnant women: reasons for admission and pregnancy characteristics eight U.S. health care centers, March 1-May 30, 2020. MMWR Morb Mortal Wkly Rep 2020;69:1355-9.

37. Pirjani R, Hosseini R, Soori T, et al. Maternal and neonatal outcomes in COVID19 infected pregnancies: a prospective cohort study. J Travel Med 2020;27: taaa158. doi: 10.1093/jtm/taaa158.

38. Vivanti AJ, Mattern J, Vauloup-Fellous C, et al. Retrospective description of pregnant women infected with severe acute respiratory syndrome coronavirus 2, France. Emerg Infect Dis 2020;26:2069-76.

39. Wang MJ, Schapero M, Iverson R, et al. Obstetric hemorrhage risk associated with novel COVID-19 diagnosis from a single-institution cohort in the United States. Am J Perinatol 2020;37:1411-6.

40. Zhang L, Jiang Y, Wei M, et al. Analysis of the pregnancy outcomes in pregnant women with COVID-19 in Hubei Province. Zhonghua Fu Chan Ke Za Zhi 2020; 55:166-71.

41. Patberg ET, Adams T, Rekawek P, et al. COVID-19 infection and placental histopathology in women delivering at term. Am J Obstet Gynecol 2020 Oct. 19 [cyberpublication avant impression];S0002-9378(20)31194-7. doi: 10.1016/j. ajog.2020.10.020

42. Yang R, Mei H, Zheng T, et al. Pregnant women with COVID-19 and risk of adverse birth outcomes and maternal-fetal vertical transmission: a population-based cohort study in Wuhan, China. BMC Med 2020;18:330.

43. Ahlberg M, Neovius M, Saltvedt S, et al. Association of SARS-CoV-2 test status and pregnancy outcomes. JAMA 2020;324:1782.

44. Cunarro-Lopez Y, Cano-Valderrama Ó, Pintado-Recarte P, et al. Maternal and perinatal outcomes in patients with suspected COVID-19 and their relationship with a negative RT-PCR result. J Clin Med 2020;9:3552.

45. Rios-Silva M, Murillo-Zamora E, Mendoza-Cano O, et al. COVID-19 mortality among pregnant women in Mexico: a retrospective cohort study. J Glob Health 2020;10:020512.

46. Woodworth KR. Birth and infant outcomes following laboratory-confirmed SARS-CoV-2 infection in pregnancy - SET-NET, 16 jurisdictions, March 29October 14, 2020. MMWR Morb Mortal Wkly Rep 2021;69:1635-40.

47. Yazihan N, Tanacan A, Erol SA, et al. Comparison of VEGF-A values between pregnant women with COVID-19 and healthy pregnancies and its association with composite adverse outcomes. J Med Virol 2021;93:2204-9.
48. Adhikari EH, Moreno W, Zofkie AC, et al. Pregnancy outcomes among women with and without severe acute respiratory syndrome coronavirus 2 infection. JAMA Netw Open 2020;3:e2029256.

49. Edlow AG, Li JZ, Collier AY, et al. Assessment of maternal and neonatal SARS CoV-2 viral load, transplacental antibody transfer, and placental pathology in pregnancies during the COVID-19 pandemic. JAMA Netw Open 2020;3:e2030455.

50. Erol SA, Tanacan A, Anuk AT, et al. Evaluation of maternal serum afamin and vitamin E levels in pregnant women with COVID-19 and its association with composite adverse perinatal outcomes. J Med Virol 2021;93:2350-8.

51. Hcini N, Maamri F, Picone O, et al. Maternal, fetal and neonatal outcomes of large series of SARS-CoV-2 positive pregnancies in peripartum period: a single-center prospective comparative study. Eur J Obstet Gynecol Reprod Biol 2021;257:11-8.

52. Jenabi E, Bashirian S, Khazaei S, et al. Pregnancy outcomes among symptomatic and asymptomatic women infected with COVID-19 in the west of Iran: a case-control study. J Matern Fetal Neonatal Med 2020 Dec. 15 [cyberpublication avant impression];1-3. doi: 10.1080/14767058.2020.1861599.

53. Jering KS, Claggett BL, Cunningham JW, et al. Clinical characteristics and outcomes of hospitalized women giving birth with and without COVID-19. JAMA Intern Med 2021 Jan. 15 [cyberpublication avant impression];e209241. doi: 10.1001/jamainternmed.2020.9241.

54. Pineles BL, Alamo IC, Farooq N, et al. Racial-ethnic disparities and pregnancy outcomes in SARS-CoV-2 infection in a universally tested cohort in Houston, Texas. Eur J Obstet Gynecol Reprod Biol 2020;254:329-30.

55. Di Mascio D, Khalil A, Saccone G, et al. Outcome of coronavirus spectrum infec tions (SARS, MERS, COVID-19) during pregnancy: a systematic review and meta-analysis. Am J Obstet Gynecol MFM 2020;2:100107.

56. Matar R, Alrahmani L, Monzer N, et al. Clinical presentation and outcomes of pregnant women with COVID-19: a systematic review and meta-analysis. Clin Infect Dis 2021;72:521-33.

57. Gheblawi M, Wang K, Viveiros A, et al. Angiotensin-converting enzyme 2: SARS CoV-2 receptor and regulator of the renin-angiotensin system. Circ Res 2020;126:1456-74

58. Coronado-Arroyo JC, Concepción-Zavaleta MJ, Zavaleta-Gutiérrez FE, et al. Is COVID-19 a risk factor for severe preeclampsia? Hospital experience in a developing country. Eur J Obstet Gynecol Reprod Biol 2021;256:502-3.

59. Todros T, Masturzo B, Francia SD. COVID-19 infection: ACE2, pregnancy and preeclampsia. Eur J Obstet Gynecol Reprod Biol 2020;253:330.

60. Been JV, Ochoa LB, Bertens LCM, et al. Impact of COVID-19 mitigation measures on the incidence of preterm birth: a national quasi-experimental study. Lancet Public Health 2020;5:e604-11.

\section{Intérêts concurrents : Aucun déclaré.}

Cet article a été révisé par des pairs.

Affiliations : Département d'obstétrique-gynécologie (Wei), Centre hospitalier universitaire Sainte-Justine; Centre de recherche du Centre hospitalier de l'Université de Montréal, Département de médecine sociale et préventive (Auger), École de santé publique, Université de Montréal; Bureau d'information et d'études en santé des populations (Wei, Bilodeau-Bertrand, Auger), Institut national de santé publique du Québec, Montréal, Qc; Centre de surveillance et de recherche appliquée (Liu), Agence de la santé publique du Canada, Ottawa, Ont.

Collaborateurs : Shu Qin Wei et Nathalie Auger ont contribué à la conception de l'étude. Shu Qin Wei et Marianne Bilodeau-Bertrand ont examiné les articles et évalué la qualité des études. Shu Qin Wei et Shiliang Liu ont effectué l'extraction et l'analyse des données. Shu Qin Wei a rédigé le manuscrit, et Nathalie Auger, Marianne Bilodeau-Bertrand et Shiliang Liu l'ont révisé de manière critique pour le contenu intellectuel important. Tous les auteurs ont contribué à l'interprétation des données, ont donné leur approbation finale pour la version destinée à être publiée et assument l'entière responsabilité de tous les aspects du travail.

Financement : Ce travail a été financé par une bourse des Instituts de recherche en santé du Canada ( $n^{\circ}$ PJT-162300). Nathalie Auger a reçu une bourse de carrière du Fonds de recherche du Québec Santé (n³4695).

Propriété intellectuelle du contenu : Il s'agit d'un article en libre accès distribué conformément aux modalités de la licence Creative Commons Attributions (CC BY-NC-ND 4.0), qui permet l'utilisation, la diffusion et la reproduction dans tout médium à la condition que la publication originale soit adéquatement citée, que l'utilisation se fasse à des fins non commerciales (c.-à-d., recherche ou éducation) et qu'aucune modification ni adaptation n'y soit apportée. Voir : https://creativecommons.org/licenses/by-nc-nd/4.0/deed.fr.

Partage des données: Toutes les données pertinentes sont fournies dans l'article et les annexes, et sont accessibles pour utilisation.

Accepté : le 2 mars 2021

Correspondance : Nathalie Auger, nathalie.auger@inspq.qc.ca 